\title{
Atomistic Design of High Strength Crystalline-Amorphous Nanocomposites
}

\author{
Shin Yamamoto ${ }^{1}$, Yun-Jiang Wang ${ }^{2, *}$, Akio Ishii ${ }^{1}$ and Shigenobu Ogata ${ }^{1,2, *}$ \\ ${ }^{1}$ Department of Mechanical Science and Bioengineering, Graduate School of Engineering Science, Osaka University, \\ Osaka 560-8531, Japan \\ ${ }^{2}$ Center for Elements Strategy Initiative for Structural Materials (ESISM), Kyoto University, Sakyo, Kyoto 606-8501, Japan
}

\begin{abstract}
There is a long-standing demand for materials which could simultaneously demonstrate multiple promising properties like high strength, good ductility and toughness. In this study, a three-dimensional bulk nanocomposite material which is composed of nanoscale crystalline metal and metallic glass is revealed to present high strength and potentially good ductility by molecular dynamics. A critical high strength is achieved by varying the ratio between crystalline and amorphous phase. The critical strength is revealed to be higher than that expected from the rule of mixture. The mechanism underlying the occurrence of critical strength in the nanocomposite is elucidated by the interaction between dislocation and matrix of amorphous phase. Our concept could guide the engineers to design more advanced bulk nanostructured materials. [doi:10.2320/matertrans.MH201316]
\end{abstract}

(Received March 1, 2013; Accepted April 5, 2013; Published May 24, 2013)

Keywords: nanocomposite, strength, ductility, molecular dynamics

\section{Introduction}

Searching for materials with excellent compromises among multiple properties such as strength, ductility, toughness, thermal and electric conductivities has been the focus of recent studies in material science and engineering for several decades. ${ }^{1,2)}$ Great achievements have been reached by either reducing the sample size of materials, or crystallite size of polycrystal to nanoscale, e.g., smaller than $100 \mathrm{~nm}^{3)}$ Those bulk nanostructured metals (BNMs) generally present very high strength in contrast with their singlecrystalline, or coarse-grained counterparts. ${ }^{4}$ Grain boundaries impede the movement of dislocations, and therefore changing grain size could influence the strength of such BNMs. ${ }^{2)}$ It is a mechanism of grain boundary strengthening which is well-known as the Hall-Petch relationship. ${ }^{5,6)}$ However, the deformability of such nanoscale materials are generally quite limited, which restricts their applications in various conditions. Polycrystal becomes amorphous material, such as metallic glass (MG), as their grain size approaches to the lower limit. MG is a kind of materials with superior mechanical properties besides great wear and corrosion resistance, and other promising functions. But poor ductility is also one of the biggest issues unsolved for MG. Localized shear deformation (shear band) is frequently observed as the failure mode of MGs, which substantially limits their applications as structural materials. To solve the brittle nature of both BNMs and MGs, nanolaminates with alternating layers of nanoscale MGs and crystalline metals have been recently synthesized. ${ }^{7,8)}$ The new category of nanolaminates utilize the concept that MGs demonstrate homogenous plasticity instead of catastrophic failure when their size is extremely small. ${ }^{8)}$ Therefore, they could exhibit apparent tensile ductility. By varying the size of its compositions, the nanolaminates could demonstrate higher strength than that of both MGs and nanocrystalline

*Corresponding author, E-mail: yjwang@me.es.osaka-u.ac.jp, ogata@ me.es.osaka-u.ac.jp metals. The improved strength and ductility are furthermore suggested due to a transition of deformation mechanisms from localized plasticity to more homogenous extension as the size decreases. ${ }^{8)}$

Here we use atomistic modeling to optimize the mechanical responses of a kind of three-dimensional (3D) nanocomposite materials which are composed of nanoscale crystalline copper and $\mathrm{CuZr}$ metallic glass. It is actually a spatially extension of the experimentally synthesized 2D nanolaminates. ${ }^{7,8)}$ The aim of this study is to design a nanocomposite which could demonstrate higher strength as well as better ductility than that expected from the conventional rule of mixtures. The main considerations underlying the highly expected mechanical properties in the present nanocomposites could be summarized as below. (i) Reducing the length scale of crystalline metals and MGs to nanoscale could lead to high strength for both compositions. (ii) The composition of crystalline metals could provide sustainable deformability. (iii) 3D networks of crystalline phase may prohibit the formation and propagation of shear bands in MGs, avoiding catastrophic shear failure. (iv) Altering the relative size of MGs versus crystals could change the behavior of dislocation nucleation, and annihilation at the boundary heterostructure, as well as the interaction between dislocation and amorphous matrix. The role of amorphous phase as capacity sink for dislocation could be manipulated so as to search for a nanocomposite exhibiting the highest strength as well as acceptable ductility. Taking these points into consideration, we successfully predict a 3D nanocomposite with superior strength than many other counterparts, e.g., bulk crystalline copper, bulk $\mathrm{CuZr} \mathrm{MG}$, and nanocrystalline crystal with similar crystalline size. By changing the volume fraction of each components, a critical strength of the nanocomposite is optimized due to a transition of deformation mechanism involving in the interaction between dislocation and amorphous matrix. We therefore provide a possible pathway to synthesize bulk structural materials with excellent strength and ductility. 
(a)

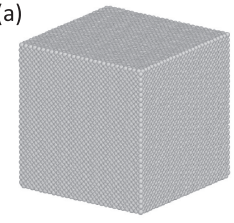

Crystal

(c)

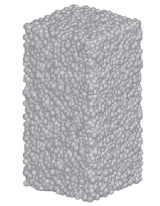

Metallic glass

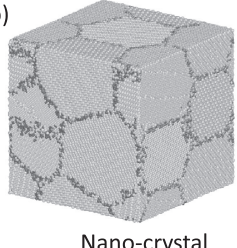

(d)

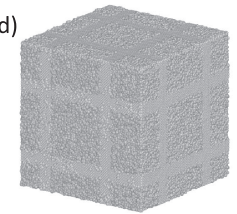

MG+crystal
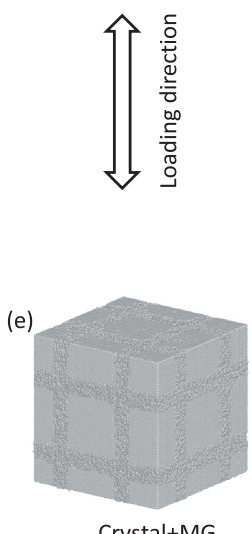

Fig. 1 Simulations models. (a) Singlecrystalline copper. (b) Nanocrystalline copper. (c) Bulk MG. (d) Nanocomposite model I: nanoscale MG cubes embedded in crystalline copper matrix. (e) Nanocomposite model II: nanoscale copper cubes embedded in $\mathrm{MG}$ matrix. Atoms in (b) are colored according to their coordination number implemented in ATOMEYE to distinguish those in grain boundaries. ${ }^{10)}$ In other models, yellow atoms represent copper, and grey zirconium. The arrow indicates the loading directions for all the samples.

\section{Models and Atomistic Simulations}

The designed 3D nanocomposites are composed of two phases. One is crystalline copper with nanosize dimension, and the other is $\mathrm{Cu}_{50} \mathrm{Zr}_{50}$ MG. The atomistic models of nanocomposites and other relative counterparts which are used to compare mechanical properties are shown in Fig. 1. These models include pure singlecrystalline copper with orientation of [100], [010] and [001] (Fig. 1(a)). Nanocrystalline copper with grain size about $8 \mathrm{~nm}$, which is generated by a Voronoi algorithm (Fig. 1(b)). The 8 grains with almost the same size are connected by random orientations; $\mathrm{CuZr}$ MGs in the form of bulk (Fig. 1(c)). Two categories of nanocomposite models are proposed as those shown in Figs. 1(d) and 1(e). The former is nanoscale MG cubes surrounded by pure copper singlecrystal. This model includes $8 \mathrm{MG}$ cubes with the same diameter of $8 \mathrm{~nm}$, while the whole simulation box size is $20 \mathrm{~nm}$ in each direction. Therefore, the volume fraction of both MG phase and crystalline phase are roughly $50 \%$, respectively. Since MG is very brittle in nature, and copper singlecrystal is intrinsically ductile, this nanocomposite resembles the microstructure of Nickel-based superalloys, where the brittle $\gamma^{\prime}$ phase is embedded in ductile $\gamma$ phase in order to present simultaneously high strength and ductile plasticity. ${ }^{11)}$ The latter is singlecrystalline copper with diameter of $8 \mathrm{~nm}$ embedded in MG nano-matrix. The volume fraction of each phase in this model is also approximately $50 \%$. The model seems to be similar to nanocrystalline copper in structure, where MG phase acts as the role of grain boundary. However, we have a freedom to control the thickness of MG phase. Therefore, this model is like nanocrystalline metals with tunable thickness of 'grain boundary'. There is plenty of space to optimize the mechanical properties of this model due to the varying role of MGs as sources, sinks, and blocks for the nucleation and motion of dislocations as that in nanocrystalline metals. ${ }^{12-14)}$
The MG phases of the nanocomposites and other models are prepared with a cooling rate of $10^{12} \mathrm{~K} / \mathrm{s}$. All the models are initially energetically relaxed, and then are thermally equilibrated for $200 \mathrm{ps}$ by a Nosé-Hoover thermostat within a $N P T$ ensemble before loadings. ${ }^{15,16)} \mathrm{N}, P, T$ denote constant number of atoms, pressure and temperature. Temperature here is controlled as $300 \mathrm{~K}$. Periodic boundary condition is applied on each direction of the models. Qualitatively predicting a model nanocomposite material with optimal mechanical properties is the main purpose of the present study. Therefore, a modified Lennard-Jones potential is used to describe the interaction between atoms. ${ }^{17)}$ In order to estimate the mechanical performance of these models, uniaxial tension with a strain rate of $10^{8} \mathrm{~s}^{-1}$ is performed to each sample. During tension, the perpendicular directions are allowed to relax according to a stress-free condition. A Parrinello-Rahman technique is adopted to control the hydrostatic pressure and stress tensor during thermal relaxation and tension. ${ }^{18)}$ The loading direction for those samples containing crystalline copper (Figs. 1(a), 1(e) and 1(f)) is invariably [001]. It guarantees a fair comparison between singlecrystalline and composite materials.

\section{Results and Discussion}

The stress-strain curves of all the model materials under uniaxial tension are summarized in Fig. 2(a). The curves gradually come to a steady-state after the strain magnitude of $12 \%$, even those models containing large volume fraction of crystalline phase. Therefore, the flow stresses of each model could be determined within the strain range of $[12 \%, 27 \%]$. The estimated flow stresses with error bar are listed in Fig. 2(b). It is noteworthy that both of the two nanocomposite models demonstrate higher flow stresses than those of all the other counterparts. In detail, the strengths of composite model I and II are predicted to be 1.87 and $1.93 \mathrm{GPa}$, respectively. They are respectively 46 and $51 \%$ higher than the strength of singlecrystalline copper, which is predicted to be $1.28 \mathrm{GPa}$. Their strengths are also higher than that of bulk MG $(1.40 \mathrm{GPa})$. Therefore, the nanocomposite models demonstrate higher strength than the value expected from conventional rule of mixture. It could be a striking feature for the possible application of this category of crystalline-amorphous nanocomposites. It is more interesting to find that the composite models do not only show better mechanical properties than that of singlecrystalline metal, but also than that of other nanostructure which is commonly thought to have high strength. For example, the strength of nanocrystalline copper with grain size of $8 \mathrm{~nm}$ (similar size to the composite models) is $1.73 \mathrm{GPa}$. In contrast, our simulations predict that the artificial nanocomposites could be potentially a class of high strength materials, which probably present even better mechanical properties than that of bulk nanocrystalline metals which are composed of nanoscale crystallites.

Between the two nanocomposite model I and II (Fig. 1), the latter demonstrates higher mechanical strength. The MG phase in composite model II acts partially as the role of grain boundary in nanocrystalline metals, which could impede 

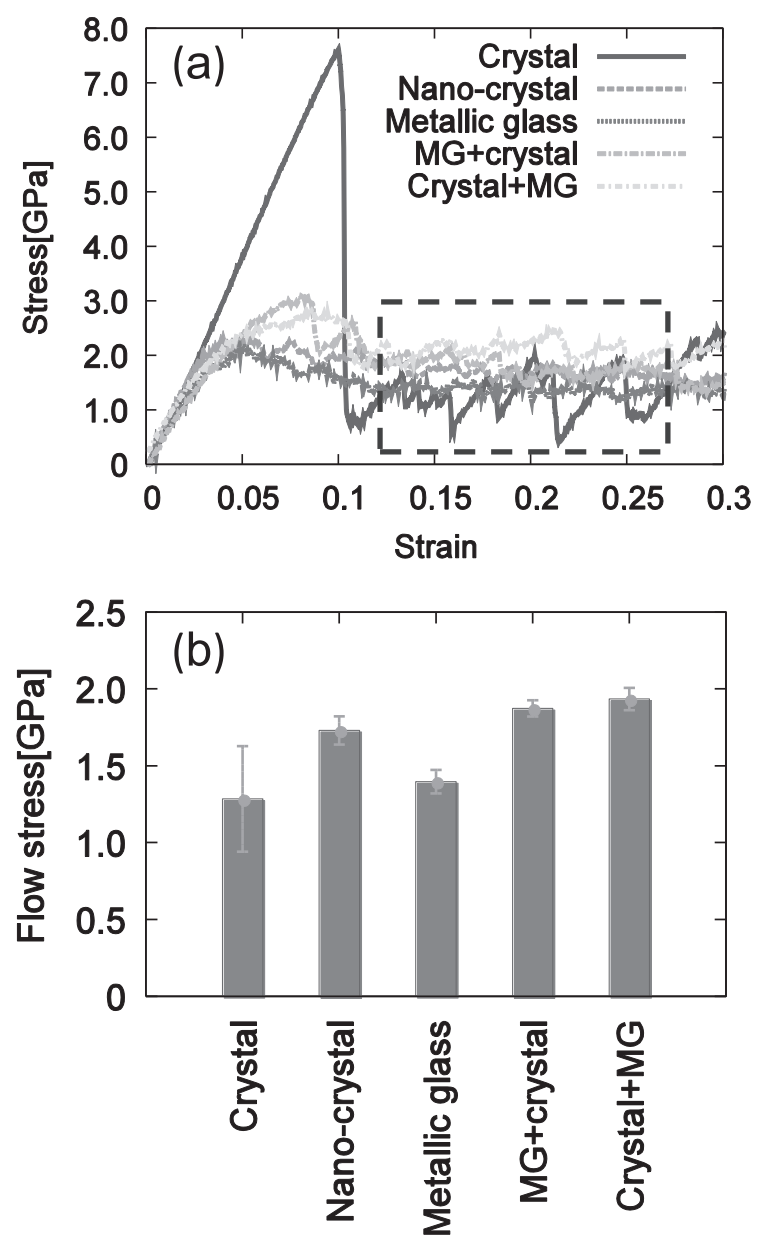

Fig. 2 (a) Stress-strain curves of different models listed in Fig. 1 under uniaxial tension. (b) Flow stresses of the samples, which are determined as the strain interval of $12-27 \%$ of the stress-strain curves.

the successive motion of dislocations, and consequently increases the strength of nanostructures. But such nanocomposites resembling BNMs are likely to be brittle. However, we have an opportunity to improve the deformability of nanocomposites without sacrificing its strength since there exists a extra degree of freedom to modulate the width of amorphous phase (grain boundary with 'tunable' width). Besides dislocation sources and blocks, thick amorphous matrix in the nanocomposite could also serve as high-capacity sinks for dislocations, enabling the adsorption of finite strain provided by the nucleation and glide of dislocations. Once the amount of amorphous phase is varied, its versatile roles could be adjusted via different ways of interactions with dislocations. Later in this study, we will illustrate MG phase could be either full, or partial blocks of dislocations.

Then we focus on optimizing a best performance of strength in nanocomposite model II. Here we fix the simulation cell to be $20 \mathrm{~nm}$ on each direction, which contains 8 units of crystalline copper cubes plus MG matrix. Each unit has the same size of about $10 \mathrm{~nm}$. The volume fraction of crystalline phase is controlled from 0 to $100 \%$. The lower and upper limits of the nanocomposite are actually pure singlecrystalline copper and pure $\mathrm{CuZr}$ MG. The obtained flow stress of model II as a function of volume fraction of

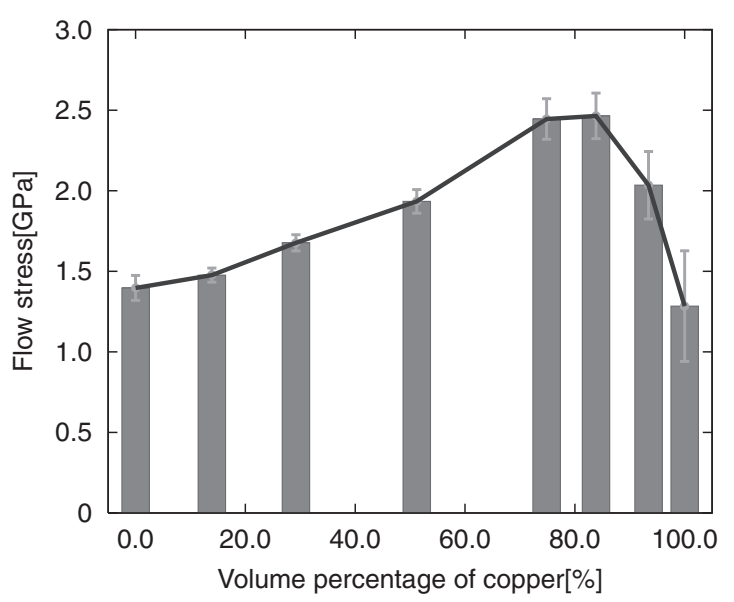

Fig. 3 Flow stress of nanocomposite model II as a function of volume fraction of crystalline copper.

crystalline phase is plotted as Fig. 3. Once more we find the composite model demonstrates higher strength than those of both crystalline copper and $\mathrm{MG}$ regardless the ratio between the two compositions. The flow stress initially increases, and then decreases with the increasing volume fraction of crystalline phase. The critical volume fraction is about $84 \%$. The appearance of a critical strength in the nanocomposite is appealing since one could adjust the mechanical performance of this material so as to meet different conditions of their potential applications. The strongest strength is estimated to be $2.46 \mathrm{GPa}$, which is about $92 \%$ higher than that of pure crystalline copper, and $75 \%$ higher than that of pure MG. The critical strength is corresponding to the composite model where the size of crystalline phase is about $9.4 \mathrm{~nm}$, and therefore the width of MG phase is about $0.6 \mathrm{~nm}$. The latter is near to the common idea of grain boundary width of nanocrystalline metals. ${ }^{19)}$

What is the mechanism underlying the transition of strength in the nanocomposite model? The different role that MG phase plays in the motion of dislocations should be a possible solution. In Fig. 4 we list two typical snapshots of nanocomposites during deformation. We consider here two situations where the thickness of MG phase are 3 and $0.5 \mathrm{~nm}$, respectively. The former is within the strengthening mode, and the latter is within the scope of softening mode as shown in Fig. 3. A common feature of the deformation mechanism in the nanocomposite is dislocation nucleation from the interface between two compositional phases. Thus the interface actually acts as a source for dislocations. Since the size of the present nanostructure is about $10 \mathrm{~nm}$, we do not have a chance to notice a full dislocation of copper, in which the leading and trailing dislocation maybe separated from several tens of nanometers. The leading partial dislocations propagate across the whole crystalline copper freely before touching another interface which is ahead of the propagation direction. However, the interaction between dislocations and MG phase could be different when the thickness of the latter is within strengthening, or softening regimes shown in Fig. 3. When the MG is thick, it could effectively impede successive travel of dislocation from one grain to the other (Fig. 4(a)). This feature resembles it as the 


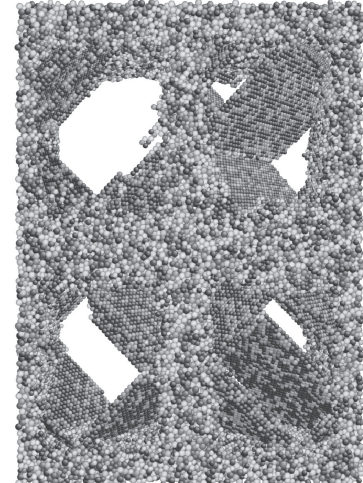

(a) MG thickness $=3.0 \mathrm{~nm}$ $(\varepsilon=0.260)$

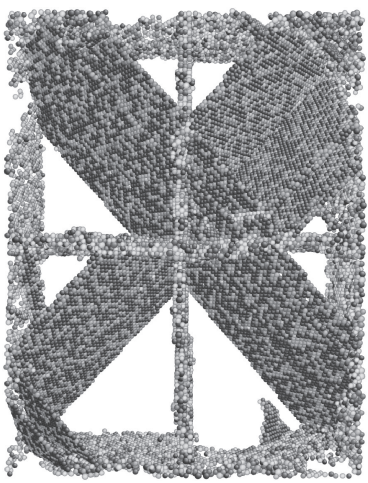

(b) MG thickness $=0.5 \mathrm{~nm}$ $(\varepsilon=0.208)$
Fig. 4 Deformation mechanisms of nanocomposite materials. (a) Dislocations nucleate from the interface between crystalline cubes and amorphous matrix, and then stops at the interface, or be adsorbed by MG phase when it is thick, which is served as a strengthening mechanisms. (b) Nucleated dislocations could penetrate the narrow amorphous matrix, which is a softening mechanism. The competition between these two deformation modes leads to a critical strength in the nanocomposite model as that shown in Fig. 3. Atoms are colored according to their centrosymmetry parameter which is implemented in ATOMEYE. ${ }^{10)}$

role of grain boundary in polycrystals. However, we find here that the MG can adsorb the existing dislocations, and subsequently opens cleaner field which is ready for the new dislocation nucleation. Then the material could provide sustainable strain in nanoscale during deformation, in keeping with high flow stress which is necessary for the dislocation nucleation. Therefore, thicker MG layer could be termed as 'terminator and cleaner' of dislocation. The new concept here is similar to the coherent twins in the recently proposed nanotwinned metals with high strength as well as good ductility. ${ }^{20-22)}$ On the other hand, the dislocations can sometimes penetrate the thin MG layer when it is smaller than $0.6 \mathrm{~nm}$. Therefore, MG phase is no more strong strengthening factor in terms of the dislocation propagation. As a result, the strength of composite material approaches to that of a singlecrystalline copper, which is much lower. Finally, we note that there is no shear banding happens in the deformation of MG counterpart in the present composite models. However, the absence of nanoscale local shear bands does not directly evidence the macroscopic ductility, which should be carefully discussed in view of not only intrinsic ductility of materials, but also specimen size and characteristic lengths of material structure. This will be done in our future work using multiscale modeling.

We could explain the strengthening and softening modes discovered in the flow stress of the nanocomposite (see Fig. 3) in terms of different interaction between MG matrix and dislocations. As the volume fraction of crystalline phase increases, the absolute size of crystalline copper increases since we have fixed the volume of unit cube to be $10^{3} \mathrm{~nm}$. The nanocomposite with crystalline phase smaller than $84 \%$ resembles nanocrystalline copper, and MG phase could be regarded as grain boundary. The amorphous matrix can also absorb dislocation in crystalline phase, which needs stress to nucleate new dislocation during deformation. It is wellknown that nanocrystalline metals with grain size $10 \mathrm{~nm}$ stay well in the regime of reverse Hall-Petch relation. ${ }^{1,2)}$ As a result, its strength increases with the volume fraction of crystalline phase, which is equal to increasing grain size. However, this relation breaks down when the MG phase is too thin to block the propagation of dislocations. Transition of deformation mechanism occurs when the MG is thinner than $0.6 \mathrm{~nm}$. Then the grain boundary strengthening mechanism becomes softening mechanism. The strength of nanocomposite approaches to that of bulk copper, which is much lower as revealed by our atomistic modelings.

\section{Summary}

In summary, a 3D nanocomposite is predicted to be a potentially high strength material. This group of bulk nanocomposite materials are composed of nanoscale crystalline copper which is embedded in nanosize matrix of $\mathrm{CuZr}$ MG. The flow stress of the nanocomposite could be as high as $2.46 \mathrm{GPa}$ at the strain rate of molecular dynamics level. The strength of this promising material is about 92,75 and $42 \%$ higher than that of singlecrystalline copper, bulk $\mathrm{CuZr} \mathrm{MG}$, and nanocrystalline copper, respectively, at the same loading rate. The thickness of MG matrix could be modulated so as to either fully, or partially impede the successive movement of dislocations. Thick MG phase can adsorb partial dislocations within the crystalline phase and provide cleaner grain, which is strengthening mechanism. However, nucleated dislocation may penetrate the MG phase when it is thinner than $0.6 \mathrm{~nm}$, which is softening mechanism. The competition between the strengthening and softening role played by the MG phase in this nanocomposite leads to a strongest strength when the volume fraction of crystalline copper reaches about $84 \%$. Although the present nanocomposite is composed of crystalline copper and $\mathrm{CuZr}$ MG, we do not employ any specific features of these two phases. Therefore, our concept could be general to nanocomposites which are composed of other metals and amorphous phases. We note that the nanocomposite is potentially to be a ductile material. The finite strain generated by the nucleation and slip of dislocations in crystalline phase could be compromised by the shear transformation zones which occur in the MG phase. ${ }^{6)}$ Therefore, it is possible to observe a homogenous deformation in this composite instead of catastrophic localized shear deformation as that happens in MGs and brittle failure of nanocrystalline metals. More efforts from both experiments and modelings are needed to clarify the potentially ductile nature of the proposed promising crystalline-amorphous nanocomposite, in particular to understand the interaction between dislocation and the amorphous phase.

\section{Acknowledgments}

This work is partially supported by the Scientific Research on Innovative Area "Bulk Nanostructured Metals" (No. 22102003), Elements Strategy Initiative for Structural Materials (ESISM), Scientific Research (A) (No. 23246025), Challenging Exploratory Research (No. 22656030), and JST under Collaborative Research Based on Industrial Demand (Heterogeneous Structure Control). 


\section{REFERENCES}

1) M. A. Meyers, A. Mishra and D. J. Benson: Prog. Mater. Sci. 51 (2006) 427.

2) T. Zhu and J. Li: Prog. Mater. Sci. 55 (2010) 710.

3) C. C. Koch, I. A. Ovid'ko, S. Seal and S. Veprek: Structural Nanocrystalline Materials: Fundamentals and Applications, (Cambridge University Press, Cambridge, 2007).

4) X. X. Huang, N. Hansen and N. Tsuji: Science 312 (2006) 249

5) E. O. Hall: Proc. Phys. Soc. London B 64 (1951) 747.

6) N. J. Petch: J. Iron Steel Inst. 174 (1953) 25.

7) Y. M. Wang, J. Li, A. V. Hamza and T. W. Barbee, Jr.: Proc. Natl. Acad. Sci. U.S.A. 104 (2007) 11155.

8) J. Y. Kim, D. Jang and J. R. Greer: Adv. Funct. Mater. 21 (2011) 4550.

9) D. C. Jang and J. R. Greer: Nat. Mater. 9 (2010) 215.

10) J. Li: Model. Simul. Mater. Sci. Eng. 11 (2003) 173
11) R. C. Reed: The Superalloys: Fundamentals and Applications, (Cambridge University Press, New York, 2006).

12) Y. J. Wang, A. Ishii and S. Ogata: Phys. Rev. B 84 (2011) 224102

13) Y. J. Wang, A. Ishii and S. Ogata: Maters. Trans. 53 (2012) 156.

14) Y. J. Wang, A. Ishii and S. Ogata: Acta Mater. 61 (2013) 3866.

15) S. Nosé: Mol. Phys. 52 (1984) 255.

16) W. G. Hoover: Phys. Rev. A 31 (1985) 1695.

17) A. C. Lund and C. A. Schuh: Acta Mater. 51 (2003) 5399

18) M. Parrinello and A. Rahman: J. Appl. Phys. 52 (1981) 7182.

19) E. A. Stern, R. W. Siegel, M. Newville, P. G. Sanders and D. Haskel: Phys. Rev. Lett. 75 (1995) 3874.

20) L. Lu, Y. F. Shen, X. H. Chen, L. H. Qian and K. Lu: Science 304 (2004) 422.

21) L. Lu, X. Chen, X. Huang and K. Lu: Science 323 (2009) 607.

22) T. Zhu, J. Li, A. Samanta, H. G. Kim and S. Suresh: Proc. Natl. Acad. Sci. U.S.A. 104 (2007) 3031. 